\title{
LES EFFETS D'UN EXIL INSTITUÉ : À PROPOS DES ENFANTS RÉUNIONNAIS TRANSPLANTÉS EN MÉTROPOLE
}

\author{
MARION FELDMAN ${ }^{1}$
}

\author{
LES EFFETS D’UN EXIL INSTITUÉ : À PROPOS DES ENFANTS RÉUNIONNAIS \\ TRANSPLANTÉS EN MÉTROPOLE
}

Cette étude montre les traces des traumatismes chez des adultes nés sur l'île de la Réunion entre 1957 et 1970, âgés aujourd'hui entre 45 et 60 ans, qui ont vécu un exil institué par la politique de Michel Debré entre 1963 et 1984 : la transplantation de 2015 enfants de l'Île de la Réunion vers la Métropole. Cet exil s'est appuyé sur les institutions dont celle de la protection de l'enfance de l'époque : la DDASS (Direction départementale des affaires sociales et sanitaires). A partir d'entretiens de recherche, cet article montre que ces enfants réunionnais ont vécu un abus de filiation, via des "traumatismes cumulatifs ", abus toujours actif aujourd'hui dans la mesure où l'État français n'a pas encore reconnu la souffrance de ces enfants, souffrance induite par une opération politique. Ces Réunionnais présentent un certain nombre de troubles psychiques liés à la désaffiliation brutale et à leur vécu abandonnique, souvent aggravés par des faits de maltraitance. Leur identité encore suspendue est difficile voire impossible à assumer, et ces difficultés se répercutent sur leur descendance.

Mots-clés : enfants, Île de la Réunion, transplantation, politique, DDASS, traumatismes.

THE EFFECTS OF INSTITUTIONALLY ENFORCED EXILE: ON THE CHILDREN OF RÉUNION RELOCATED TO FRANCE

This study reveals the traces of trauma in adults born on the island of Réunion between 1957 and 1970, now aged between 45 and 60 years old and who, between 1963 and 1982, were exiled under the policy of Michel Debré which saw 2150 children relocated from Réunion to France. This exile relied upon the cooperation of institutions, including the institution responsible for child protection at the time, the DDASS (Direction départementale des affaires sociales et sanitaires, Departmental Directorate of Health and Social Affairs). On the basis of research interviews, the article shows how these children of Réunion are survivors of child abuse via "cumulative trauma"-an abuse that continues to this day in so far as the French state still refuses to recognize their suffering, a suffering brought about by an operation that was political in nature. These children from Réunion present a certain number of mental disorders rooted in their brutal removal from their families and their experience of abandonment, often aggravated by mistreatment. Their identity, still suspended, is difficult or even impossible for them to assume, and these difficulties have repercussions for their descendants.

Keywords: Children, Réunion, relocation, politics, DDASS, trauma.

1. Professeure de psychopathologie psychanalytique à l'université Paris-Nanterre. Chercheure au laboratoire "Clinique, psychanalyse, développement " ClipsyD EA4430 (A2P). Psychologue-clinicienne. 
Este estudio presenta las consecuencias del traumatismo sufrido por los adultos nacidos en La Reunión desplazados a Francia entre 1957 y 1970 y que hoy tienen entre 45 y 60 años. Este exilio institucional fue instituido por Michel Debray entre 1963 y 1984 y la DDASS (Dirección Departamento Asunto Sociales y Sanitarios) aseguró la protección de la infancia en aquella época. Una investigación basada en entrevistas con los adultos, demuestra que los niños de La Reunión sufrieron "traumatismos acumulados" respecto a su filiación. Este abuso sigue activo actualmente ya que el Estado Francés no reconoce el sufrimiento de lo niños víctimas de un dispositivo politico. Estas personas de La Reunión padeces distintos trastornos psíquicos al haberles arrebatado brutalmente su filiación, lo que vivieron como un abandono, acrecentado por episodios de maltrato. No han podido asumir una verdadera identidad y sus dificultades repercuten en sus descendientes.

Palabras clave: niños, Isla de La Reunión, desplazamiento, política, traumatismos, DASS.

La Réunion est une île de l'ouest de l'Océan Indien, à presque dix mille kilomètres de la Métropole, colonisée par la France au $18^{\mathrm{e}}$ siècle. Elle a connu différents noms : Île Bourbon, Île de la Réunion, Île Bonaparte, puis de nouveau Île de la Réunion. La population de l'île s'est " constituée sur une période de trois cents ans à partir de groupes ethniques d'origines très variées [...]. Venus de France, mais aussi de Madagascar, d'Afrique, des Indes, de Chine, des Comores, avec leurs cultures, leurs croyances, leurs langues, ces citoyens français forment [...] une véritable "mosaïque" [...]" (Payet, 2001, p.179). Ainsi, la population réunionnaise issue de la migration a été contrainte à une adaptation pour intégrer et s'intégrer, elle a ainsi vécu des multiples deuils et renoncements. À cela, s'ajoutent les problématiques de la colonisation et de l'esclavage jusqu'en 1848. Chaque famille réunionnaise a donc une histoire complexe et a été ébranlée dans son historicité. Chaque famille a dû faire avec son héritage particulier, ses représentations culturelles et son système de parenté, "entre autre également, dans tout ce qui a trait à la nomination des personnes (composition, attribution, transmission et fonction du nom)" (Ibid, p.180).

\section{UN CONTEXTE HISTORIQUE, POLITIQUE ET INSTITUTIONNEL PARTICULIER}

Entre 1963 et 1984, nous savons aujourd'hui que 2015 enfants $^{2}$ ont été transplantés de la Réunion vers la Métropole. Ce transfert se situe dans

2. Point d'étape-communication verbatim de Philippe Vitale, Président de la commission nationale d'experts, (Ministère de l'Outre-Mer, 16/02/2017). Jusqu'à très récemment, on pensait qu'il s'agissait de 1615 enfants. Lors de la remise du rapport par la Commission le 10 avril 2018 (après que ce manuscrit a été soumis à la revue), il a été précisé qu'il s'agit en fait de 2015 enfants transplantés entre 1963 et 1984. Le rapport d'expertise met en évidence le fait que cette transplantation s'est déroulée dans le cadre des pratiques et règlements qui régissaient l'institution de la protection de l'enfance à ce moment-là. Ainsi, aucun élément 
la période au cours de laquelle Michel Debré est député de La Réunion où il y est élu le 5 mai 1963. Ce choix de se présenter à La Réunion s'explique notamment par la crainte de Michel Debré de voir ce qui reste de l'empire colonial français suivre la voie empruntée par l'Algérie, une indépendance pour laquelle il est personnellement opposé. Son projet est notamment de contrer l'emprise des communistes et de se poser garant d'un pouvoir national fort.

Dans les années 1960, La Réunion connaît une situation économique et sociale grave. Le chômage en 1967 est à hauteur de plus de 13\%. L'île connaît une explosion démographique, un manque de qualification des hommes et un déséquilibre important à l'échelle des salaires (Ascaride et al., 2004, p.49). Dans ces mêmes années, les régions françaises sont inégales en matière de modernisation. Le Limousin, par exemple, est un territoire rural important qui "situé à l'écart des grands axes de circulation, est enclavé par une barrière montagneuse rendant les communications particulièrement difficiles. Le département de la Creuse appartient à cette "France du vide" à l'écart des échanges et du changement social du "baby-boom". Il faudra attendre le schéma autoroutier de 1987 pour sortir le Massif central et sa périphérie de son enclavement. Le Limousin est marqué par un affaiblissement démographique auquel s'ajoute un vieillissement généralisé de sa population " (ibid, p.118). En 1968, dans cette région, on recense $81 \%$ de ruraux alors que la France en compte $56 \%$ à la même époque (ibid, p.121).

Face à ce constat - explosion démographique d'une part et exode rural d'autre part - Michel Debré met en place une stratégie inédite de vase communicant : une politique de migration vers la Métropole. Et c'est dans ce département de la Creuse principalement ainsi que vers quatre-vingtdeux autres départements que les enfants réunionnais vont être transférés. Les institutions vont être les relais de cette politique : des campagnes de publicité vantent l'Eldorado de la France métropolitaine, avec le slogan suivant : "N'ayez pas peur de quitter l'île, on va vous aider. "

Cette politique migratoire s'appuie principalement sur l'institution de la protection de l'enfance : l'Assistance publique jusqu'en 1964 puis les Directions Départementales des Affaires Sociales et Sanitaires (DDASS) ${ }^{3}$. Ces deux institutions sont donc, l'une après l'autre, les chevilles ouvrières de la politique de Michel Debré. Le principe fondamental de l'Assistance publique et des DDASS repose alors sur la rupture des liens parentsenfants. Partant du postulat que le placement des enfants est notamment basé sur une " radiographie de la parentalité déficiente " (Jablonka, 2006, p.39), il est nécessaire de leur éviter une transmission de la dégénérescence parentale. Cette rupture familiale est notamment rendue opérante par le principe du secret des origines et du lieu de placement. La famille

valant preuve de vol d'enfant, d'enlèvement n'a été trouvé dans les dossiers. Cependant, cette commission n'avait pas mission d'évaluer les répercussions psychologiques de cette transplantation et d'ailleurs elle était composée d'un sociologue, d'un historien et d'un géographe. Il n'y avait pas de psychologue. D'autre part, soulignons que les faits recueillis dans ce rapport, la plupart issus des archives, ne viennent en aucun cas remettre en cause la subjectivité, c'est-à-dire le vécu de cette transplantation chez ceux qui l'ont éprouvée.

3. En 1985, l'institution prendra le nom d'"Aide sociale à l'enfance ". 
de l'enfant est oubliée : "C'est ainsi que l'article 69 du Code de la famille et de l'aide sociale a prévu que "le lieu de placement du pupille restait secret, sauf décision contraire du tuteur prise dans l'intérêt de l'enfant". Cette règle concernait les pupilles, mais certains services l'ont appliquée, il y a peu d'années encore, pour l'ensemble des enfants en garde et recueillis temporairement, en alléguant qu'ils étaient assimilés aux pupilles d'État " (Verdier, 1978, p.22). Il a fallu attendre l'année 1976 pour qu'une circulaire du Ministère de la Santé suggère que les relations parents-enfants sont un droit et un devoir. La loi du 6 juin 1984 l'a rappelé à nouveau.

Par ailleurs, l'aspect important qui participe de la doctrine de ces institutions de protection de l'enfance est le suivant : la misère des parents est considérée comme douteuse, et c'est le milieu rural qui reste, aux yeux des responsables, le milieu privilégié pour un meilleur épanouissement des enfants. L'institution de la protection de l'enfance est donc le pourvoyeur d'enfants capable d'endiguer l'explosion démographique d'une part et de lutter contre la désertification des campagnes d'autre part ${ }^{4}$.

Le processus à l'œuvre est de rechercher des enfants qui se situent dans les quartiers pauvres de l'île que les assistantes sociales investissent, en y repérant les familles fragilisées par la précarité avec un nombre d'enfants élevé, auxquelles elles proposent un placement dans un foyer pour un ou plusieurs de leurs enfants. Après cette séparation initiale, la DDASS de la Réunion fait pression sur les parents souvent illettrés pour qu'ils autorisent le départ de leurs enfants vers la France métropolitaine. Les parents pensaient que leurs enfants seraient recueillis temporairement, or ils signaient la séparation définitive d'avec leurs enfants et ces derniers devenaient Pupilles d'État (Ascaride et al., 2004). Les familles font confiance à l'État. Confiance car elles pensent que les enfants partent pour un court séjour alors qu'en fait il s'agit d'un aller simple. Il est à noter qu'une ordonnance du 15 octobre 1960 mise en place par Debré stipule la clôture à toute revendication : quiconque qui faisait état d'un dysfonctionnement d'une politique d'une administration était soumis à des répressions et à la prison ou exclusion du territoire. L'exil des enfants est ainsi institué.

\section{MÉTHODOLOGIE}

La méthodologie de recherche s'appuie sur celle déjà utilisée pour ma recherche précédente concernant le vécu et le devenir d'une population, les enfants juifs cachés en France pendant la Seconde Guerre mondiale (Feldman, 2009) ayant vécu des événements collectifs impactant nécessairement chaque singularité et chaque subjectivité. L'élaboration d'un modèle clinique d'analyse m'amène ainsi à le proposer dans d'autres situations afin d'en comprendre certaines relevant d'une psychopathologie

4. Durant les décennies 1960 et 1970, l'État a officiellement organisé l'émigration de quelques 75000 Réunionnais par l'intermédiaire du BUMIDOM (Bureau pour le développement des migrations dans les départements d'outre-mer). C'est dans le cadre de cette émigration organisée que 2015 enfants ont été transférés en Métropole. 
contemporaine, telle que celle qu'ont connue les enfants réunionnais transplantés en Métropole.

Dans une première étude, l'analyse qualitative des récits avait permis de repérer ce qu'étaient devenues les traces des traumatismes à travers les âges qui se sont révélées particulièrement visibles à des âges clés de la vie (Feldman, 2008 ; 2018) : adolescence, maternité, parentalité, retraite, vieillesse, grand-parentalité... Ces âges constituent des passages qui, le plus souvent, sont des périodes de réaménagements psychiques fragilisant l'individu, ou peuvent être, au contraire, des leviers de transformation. Dans la situation des enfants réunionnais, il s'est avéré que ces mêmes périodes (adolescence, maternité et parentalité) ont été des moments particulièrement difficiles. C'est ce que nous tentons d'exposer ici. Ainsi, une proposition clinique adaptée permettrait de les aider à appréhender leur deuxième moitié de vie de façon plus apaisée. Afin d'aboutir à un tel projet, il est nécessaire d'identifier les répercussions psychologiques de ces adultes ayant vécu cette transplantation, à différents moments de leur vie. Ils sont aujourd'hui âgés entre 45 et 60 ans.

Pour ce faire, plusieurs personnes ont été rencontrées, la plupart par le biais de la Fédération des Enfants Déracinés des DROM (départements et région d'outre-mer). Il s'agit de quatre femmes et deux hommes. Ils sont nés en 1958, 1960 pour deux d'entre eux, en 1963, 1967 et 1970. Ils ont donc été transférés vers la Métropole, entre l'âge de 3 et de 9 ans ${ }^{5}$. La méthode de recherche a consisté en la réalisation d'entretiens semidirectifs enregistrés et retranscrits mot à mot. Chaque entretien a eu une durée moyenne de trois heures. L'analyse qualitative longitudinale et transversale (Moro, 1994) a été réalisée selon une approche complémentariste (Devereux, 1972) prenant en compte les éclairages psychanalytiques, anthropologiques et historiques. Est notamment abordé ici un aspect de leur trauma, partagé par tous, qui est celui d'avoir été exposés à des ruptures souvent irrémédiables des liens de filiation et d'affiliation. Ces ruptures ont été exécutées pour les besoins d'un projet politique et ont eu des répercussions sur leur construction psychique car, comme le rappelle Yolanda Gampel (2006, p.68), la réalité extérieure est inhérente à la construction de la subjectivité.

\section{VALÉRIE : «CIVILEMENT, JE SUIS NÉE DANS LA CREUSE, J'AI DEUX NOMS, DEUX PRÉNOMS, DEUX BAPTÊMES, DEUX LIEUX DE NAISSANCE »}

Valérie est née en 1963 à la Réunion. Elle s'appelait Marie-Germaine. À l'âgée de 3 ans, elle est exilée en Métropole avec toute sa fratrie, deux frères et quatre sœurs. Valérie n'a aucun souvenir de sa vie à la Réunion,

5. Trois personnes ont été exilées à 3 ans, une à 4 ans, une autre avait 7 ans et une autre avait 9 ans. 
elle n'a qu'un seul "flash ": "Je me vois, je nous vois, dans cet avion avec tout un tas d'enfants, et là je me sentais complètement perdue."

Elle arrive au foyer de Guéret. Il lui a été raconté qu'ils étaient nombreux, et comme Valérie faisait partie des plus petits, elle a dormi sur un matelas par terre dans le couloir. D'autres "flashs" lui reviennent, ils sont liés à son séjour passé dans un hôpital, soignée pour des problèmes de malnutrition : "Je me vois agrippée aux jupes des infirmières. " À la sortie de l'hôpital, elle est séparée de ses frères et sœurs, et placée dans une famille d'accueil durant quatre ans où elle subit des violences : "Le seul endroit où je me sentais protégée, c'était sous la table. Je passais beaucoup de temps sous la table. Le père me frappait. " Elle était effrayée. À certains moments, un "couple de Blancs» lui rendait visite et lui apportait des poupées et des cadeaux. Elle se souvient que la fille de la famille d'accueil lui jetait systématiquement sa poupée et ses jouets dans le puits.

À l'âge de 7 ans, l'assistante sociale vient chercher Valérie et la conduit "chez [ses] parents ". Après quelques heures de voyage qui lui ont semblé très longues, elles arrivent dans un endroit où l'attendait le couple qui venait la voir de temps en temps auparavant. La femme s'approche d'elle pour lui dire qu'elle est sa mère, et que l'homme est son père. Valérie se souvient d'avoir eu très peur et de s'être précipitée sous la table avec le chien. Elle les trouvait beaux mais ils étaient des étrangers. Elle ne comprenait pas pourquoi sa mère lui disait avec tant d'insistance être sa mère, alors que Valérie percevait bien la différence de couleur de peau : "Regarde, ton père, il est bronzé ", disait-elle pourtant. L'entrée à l'école est vécue comme étant un "enfer ". Valérie se sent à nouveau abandonnée et étant la seule à avoir la peau noire, elle subit des discriminations. À l'école, on lui dit : "Blanche neige, sale négresse, tu t'es mal lavée ce matin ?" Au collège, une élève écrit une chanson sur elle : "La p'tite négresse fait pipi dans l'eau... ». Les discriminations sont telles qu'elle refuse de manger à la cantine durant les quatre années de collège. Valérie dit avoir eu peur des hommes. Elle met d'ailleurs une année avant d'accepter que son père s'approche d'elle.

Malgré ce vécu, Valérie investit de façon importante l'école, jusqu'en classe de première au lycée où un jour, alors que sa mère lui demande d'aller chercher quelque chose dans un placard, Valérie découvre des documents lui révélant la vérité sur ses origines : "Ces papiers me disaient que $j$ 'avais un autre nom, un autre prénom... ces papiers me disaient que j'avais raison. "Valérie se rend compte qu'elle est la seule à avoir vécu dans le mensonge pendant des années, alors que son entourage connaissait sa véritable identité. Elle est traversée à ce moment-là, à la fois par une grande colère et par un soulagement. Elle vit ce moment comme " un tsunami ". Elle arrête la scolarité. Et surtout elle décide de se mettre à la recherche de sa fratrie.

Son frère aîné, qui avait 13 ans lors de son arrivée en Métropole, est le premier qu'elle rencontre. Il lui dit que les services sociaux lui avaient donné l'ordre de ne pas prendre contact avec ses frères et sœurs, c'està-dire les trois plus jeunes placés dans des familles d'accueil puis adoptés. Valérie rencontre ensuite une de ses sœurs, adoptée par une famille qui s'est mal comportée avec elle. Son autre sœur, revue par la suite, a été élevée par un couple d'agriculteurs, bienveillant, qui lui a révélé son histoire. 
Ses deux autres frères, âgés de 12 et 11 ans à leur arrivée en Métropole, avaient été placés dans un foyer. Elle se rend compte alors qu'elle les avait croisés à Guéret, "sans savoir qu'on était frères et sœurs".

Durant son adolescence, Valérie a peur des hommes. Elle ne comprend pas ce qui lui arrive quand elle a ses règles pour la première fois. Sa mère ne lui dit rien. Elle raconte ne pas avoir de lien fort avec elle. Elle la dit autoritaire. À l'âge de 19 ans, elle tombe amoureuse d'un homme, à peine plus âgé qu'elle, qui est militaire de carrière. Elle se marie avec lui et a deux enfants. Sept ans après, elle divorce car il est infidèle et violent.

Elle part vivre à Guéret. Elle travaille dans différents lieux pour ensuite entrer dans l'administration à la suite de la réussite des concours de la fonction publique en 1991. Elle élève seule ses deux enfants. Elle se dit très protectrice à leur égard, et leur camoufle la vérité concernant ses origines, "ne voulant pas faire de peine à [ses] parents ".

Quelques années plus tard, un nouveau choc advient dans sa vie, lorsqu'elle apprend le suicide d'un de ses frères, alors âgé de 32 ans. C'est un "déclic " pour elle, et elle décide de partir à la Réunion. Sa mère n'y est pas favorable, mais en 1992 elle y va avec son frère et une de ses sœurs. Arrivée sur l'île, elle dit être dans un "déni total ", elle culpabilise, traversée par un conflit de loyauté vis-à-vis de ses parents adoptifs. Sa première réaction est : "Quand je vois la pauvreté dans la famille qui me dit être ma famille, je me dis "heureusement que j'ai été en Métropole"》. Elle rentre en Métropole, et dit se sentir Creusoise. C'est à ce moment-là qu'elle découvre qu'une de ses sœurs part à la dérive, avec des problèmes d'alcoolisme qui la conduisent à faire des séjours en psychiatrie. Valérie vit une période de grande instabilité. Elle dit avoir déménagé une quinzaine de fois. Elle rencontre un homme avec lequel elle vit quelques années. Mais lorsqu'elle se retrouve enceinte, il la quitte.

À l'âge de 43 ans, elle retourne à la Réunion pour un congé bonifié. De retour en Métropole, elle fait la démarche auprès de son administration pour obtenir une mutation qui est acceptée. Elle s'installe donc sur l'île durant neuf ans, notamment pour faire des recherches approfondies sur ses racines. Mais à la Réunion, son histoire est un sujet tabou. Sa famille ne veut rien lui dire. Son parrain, ami de ses parents biologiques, refuse de lui parler. Un de ses cousins se tait également en disant : "Il faut dire la vérité quand les gens sont morts. "Un jour, elle rencontre une gramoune ${ }^{6}$, qui se met à pleurer en la voyant : "Qu'est-ce que tu ressembles à ta mère !" Mais celle-ci refuse de lui révéler quoi que ce soit. À force de détermination, Valérie découvre des fragments d'histoires, notamment celle qui concerne sa sœur qui va mal. Elle aurait été violée à l'âge de 8 ans. Sa mère se serait suicidée à l'âge de 28 ans parce qu'elle était encore enceinte.

Aujourd'hui, Valérie continue à rechercher des informations, mais les langues ne se délient toujours pas. Depuis deux ans, elle est à la retraite, elle est rentrée en Métropole après s'être mariée avec un homme, décédé quatre ans après des suites d'un cancer. Elle est engagée dans les associations pour la reconnaissance de la souffrance des ex-mineurs réunionnais 
transférés en Métropole, et elle espère retourner à la Réunion à la fin de l'année 2017 pour chercher encore. Son hypothèse est qu'elle aurait été un bébé volé. Son identité a été effacée : "Civilement, je suis née dans la Creuse, j'ai deux noms, deux prénoms, deux baptêmes, deux lieux de naissance. " Dans son dossier, il n'y a pas de document d'acte d'abandon.

Aujourd'hui, elle continue à faire des cauchemars : elle est "poursuivie ", elle "se cache ", elle "tombe dans un trou ", elle a "beau [se] cacher", elle est "toujours poursuivie». Elle a toujours peur des pièces fermées. Elle avoue avoir fait deux tentatives de suicide. La première date de l'adolescence de son fils quand il avait 15 ans. Il avait des soucis de dépendance au cannabis, à l'alcool, elle allait souvent le chercher à la gendarmerie. 15 ans correspond aussi à l'âge auquel Valérie a avoué à son fils sa véritable identité, âge qu'elle avait elle, quand elle a appris d'où elle venait. Valérie a été suivie par un psychiatre, elle a eu des traitements. La deuxième tentative de suicide date de l'époque où elle a été abandonnée par le père de son troisième enfant. Elle s'est retrouvée seule à élever ses trois enfants. Elle a connu des périodes de dépression. Et puis du fait des maltraitances subies, avec des problèmes de santé importants, elle a été considérée comme travailleur handicapé. Aujourd'hui, elle continue à être suivie par un psychologue en CMPP. Elle aide ses compatriotes à faire les démarches nécessaires pour retrouver leurs racines.

\section{EN QUOI CET EXIL EST-IL TRAUMATIQUE ? ET QUELS EN SONT LES EFFETS ?}

Les traumatismes ont été induits par une politique spécifique, qui a eu pour but de séparer les enfants des parents, de les conduire en Métropole pour y faire souche. Ces traumatismes correspondent aux effets des ruptures des liens de filiation, d'affiliation, et des ruptures avec leurs repères géographiques. L'enfant n'est plus enfant de ses parents, mais il devient d'abord enfant de l'institution en tant que "pupille d'État ", puis enfant de parents métropolitains adoptants. Il change parfois de prénom et de nom lors de l'adoption. Dans quelques situations, il est arrivé qu'il change de lieu de naissance. Dans la majorité des cas, il est séparé de sa fratrie car l'éclatement de ce sous-système familial semble voulu et organisé. Jablonka (2007) souligne ainsi que la DDASS transgresse l'article $67 \mathrm{du}$ Code de la famille et de l'aide sociale (CFAS) qui stipule que "les frères et sœurs sont placés dans la même famille et, en cas d'impossibilité, dans la même commune" (p.44). Par ailleurs, il leur est interdit de parler le créole.

Marie-Françoise, née en 1960 et conduite en Métropole à l'âge de 3 ans, raconte : "On dit pas un zavion, on dit un avion. [...] j'ai eu le droit à des coups parce que je parlais créole. Mais je savais pas que je parlais créole, il a fallu que j'apprenne, par une Réunionnaise, que je parlais créole. "

Jean-Roger, né en 1960 et arrivé en Métropole à 7 ans, dit que ses parents adoptifs lui apprenaient à oublier le créole et à parler le français, le père "à coup de baffes" ". 
Jean-Paul, né en 1970, est exilé à l'âge de 3 ans. Il dit n'avoir aucun souvenir avant l'âge de 6 ans, date à laquelle il a commencé à parler. Entre l'âge de 3 ans et 6 ans, il était mutique. Il raconte avoir bénéficié de nombreuses séances d'orthophonie et avoir été en difficulté scolaire.

Le mensonge participe activement de cette politique : les organisateurs mentent aux parents en leur promettant que leurs enfants reviendront et qu'ils feront des études en Métropole. Mensonges aux enfants aussi : avant de quitter La Réunion, il leur est montré des belles images de la France, en leur promettant de faire du ski, de fêter Noël. Parfois, avant leur départ, certains racontent avoir subi des maltraitances dans les foyers de la Réunion.

Marie-Claire, née en 1958, transférée en Métropole à l'âge de 9 ans et demi, se souvient : "Donc je me retrouve dans cet avion, complètement perdue, des enfants qui pleurent autour de moi ".

Jean-Roger se rappelle d'avoir eu très peur dans l'avion et d'avoir crié.

Quand ils arrivent en Métropole, les représentants des institutions françaises leur disent qu'ils sont orphelins ou qu'ils ont été abandonnés.

Marie-Claire poursuit : "Tu es orpheline, tu n'as plus de parents, tu n'as plus de famille, ni frère, ni sœur : on m'a dit que j'étais orpheline... Elle m'a dit : 'tu es orpheline de père et de mère", ça je vous dirai comment je l'ai su : "tu es orpheline de père et de mère, tu n'as pas de parents, tu n'as pas de frères, tu n'as pas de sœurs" ".

Jean-Paul dit que ses parents adoptifs lui ont toujours dit qu'il était originaire de la Réunion. La seule information qu'ils détenaient c'est le fait qu'il aurait été abandonné par sa mère, car elle était très jeune et très pauvre.

La séparation pour l'enfant est source d'angoisse de perte et d'abandon. Les seules paroles qui accompagnent ces séparations sont mensongères, vont durer dans le temps et sont encore actives aujourd'hui. Ainsi, les risques de dépression, de dépersonnalisation, de troubles de l'attachement sont grands. Ces ruptures sont à l'origine de la discontinuité interne avec une perte du sentiment continu d'exister, si important pour permettre la construction du Sujet. En outre, la séparation parents-enfant inclut plus largement la séparation des lieux et des odeurs qui sont familiers à un enfant. La capacité d'un enfant à garder vivante en lui la représentation d'une personne aimée est limitée lorsqu'il n'a pas l'occasion de la voir ou de lui parler. "Tout se passe bien pendant quelques jours, quelques semaines, puis, soudain, l'enfant ne sait plus si sa mère existe réellement " (Winnicott, 1984, p.68).

Soulignons le fait que l'adoption a souvent lieu à la suite de plusieurs autres séparations. Après la séparation de sa famille biologique, l'enfant est placé en foyer ou pouponnière à La Réunion. Puis arrivé en Métropole, il est souvent orienté vers un foyer ou une famille d'accueil où il peut rester quelques semaines, mois, voire plusieurs années, avant d'être adopté, comme cela a été le cas pour Valérie. L'âge de survenue des séparations est une donnée particulièrement importante. L'impact de ces ruptures et de ces mensonges va dépendre du stade de la vie psychique de l'enfant. Son développement est en effet d'autant plus dépendant de l'apport de la réalité externe qu'il est plus jeune. L'existence 
d'une bonne relation d'objet primaire, du langage, de la capacité de symbolisation est un facteur de protection.

Par ailleurs, l'Église participe à cette rupture des origines. En effet, ces enfants réunionnais sont yabs, malbars, cafres, zarabs ou chinois? ${ }^{7}$. Lorsqu'ils arrivent en Métropole, ils sont baptisés et ainsi convertis au catholicisme.

Marie-Françoise, transférée à l'âge de 3 ans dans la Creuse et adoptée trois ans plus tard, s'appelle Claude, dorénavant : "Sur la plaque qu'on m'a plantée au-dessus du lit, parce qu'il y avait l'époque des plaques avec les saints, c'était la mode avec les saints. On offrait ça aux anniversaires et l'obligation de fréquenter le catéchisme."

Marie-Claire : "La messe tous les dimanches, le catéchisme avec des curés pervers "; elle ajoute : "Même d'autres personnes que j'ai retrouvées de St Clar, qui m'ont dit la même chose."

Des humiliations, de la maltraitance, des abus sexuels avant et/ou après le voyage participent, pour beaucoup d'entre eux, des effets traumatiques de l'exil. A l'arrivée au foyer de l'enfance de Guéret dans la Creuse, certains parlent d'un accueil complètement dépersonnalisé voire désubjectivé : "On nous met nus dans une pièce, en slip, je voyais une grosse machine à vapeur et on nous a désinfectés. " Il y a un tel nombre d'enfants que des matelas sont mis dans les couloirs. "Beaucoup ont été maltraités par les familles adoptantes : utilisés comme "garçons de ferme ", ou certaines filles ont été abusées sexuellement. Aucun des sujets interrogés n'a atteint le niveau du baccalauréat.

Marie-Françoise a connu de la violence dans sa famille d'adoption : "Je disais $3 \times 2,8$, j'avais pas besoin d'une claque franchement "; ou bien : "Quand j'ai grandi, j'avais des petits copains, des copines, et quand je voyais comment ils étaient traités, je me disais : ici y a un problème, parce que là, ma copine elle a fait une bêtise, mais elle a pas reçu une claque de sa maman, elle a été réprimandée, et moi parce que je réponds 8 à la place de 12 , je casse un verre, je me retrouve avec... en plus j'ai une punition, ce qui est bien sur les peaux foncées, les bleus ça se voit pas. "

S'étant fermement opposée à trois reprises à l'adoption, Marie-Claire est restée dans le foyer où elle a été accueillie dès son arrivée en Métropole : "On m'a embauchée pendant dix ans. Et les deux premières années, on s'est servie de moi comme femme de ménage. Et ensuite, je suis restée treize ans comme éducatrice là-dedans. Et ensuite, les autres années, donc, j'ai remplacé tous les éducateurs qui partaient en formation d'éducateur spécialisé. En fait j'ai servi de bouche-trou."

Les interrogations sur l'origine sont présentes et réactivées sans cesse par les moqueries, des discriminations subies notamment en lien avec le fait que ces enfants n'ont pas la même couleur de peau que leurs camarades : "On m'appelait Chocolat, petit noireau ", dit une personne interrogée, qui a été conduite à St Clar dans le Gers.

7. Les Yabs sont les descendants des Bretons, les Malbars sont hindous ou tamouls d'origine indienne, les Cafres sont noirs d'origine africaine et esclaves, les Zarabs sont musulmans d’origine indienne ou pakistanaise. Les Français de Métropole sont les Zoreilles. 
Un bus du village passait et nous récupérait devant l'aérium de St Clar et on était considérés comme des cas sociaux, des malades mentaux [...]. J'ai pas supporté qu'on me donne ce genre de quolibet. À l'époque, on disait "négresse ". En 1969, tu arrives dans un petit village, y'a pas une seule personne de couleur.

Arrivés à l'adolescence, la vie devient particulièrement difficile. Période de réaménagements psychiques, c'est aussi une période au cours de laquelle des questions ontologiques se posent : À qui je ressemble ? À quoi j'appartiens ? Qui je veux être ? (Mansouri, 2013). Des interrogations sur les appartenances sont posées. Ce passage est vécu douloureusement. Temps de réactivation de la problématique œdipienne, le sujet adolescent est supposé désidéaliser les images parentales introjectées durant l'enfance, alors que dans le même temps s'imposent à lui les interrogations sur l'héritage familial (Ibid., 2013). Pour ces enfants, il s'agit peut-être d'une dislocation d'images parentales introjectées, notamment pour les plus petits. Ils se construisent ainsi sur des clivages, des discontinuités, des " trous " qu'il va leur falloir combler de façon parfois quasi-pulsionnelle pour acquérir un simulacre de sécurité, essentielle pour vivre, mais nécessairement accompagnée de défenses pathologiques.

Marie-Claire : «Moi quand j'avais mes règles, je comprenais pas qu'est-ce que j'avais, pourquoi ça coule " [...] "Et à chaque Noël, on se retrouvait toutes seules dans les bars. Et où sont les gens ? Bah les gens étaient tous chez eux en famille, à faire la fête. Et nous on était perdues. Et puis c'est à ce moment-là que je me suis rendu compte que quelque chose n'allait pas... dans ma vie, parce que voilà, j'ai eu un avortement, j'ai eu bien plus que ça. "

Marie-Françoise se souvient de cette période : "Je suis en 4ème, ou en 3ème. Bizarrement, je suis placée parce que je fais partie des enfants turbulents "... "Je faisais du sport, de l'athlétisme, de la compétition. Ils m'ont coupé de tout ça. " Cette période correspond également au divorce de ses parents adoptifs. "J'ai 16 ans, je tombe amoureuse... il est devenu mon beau-père. "

À partir de cette période, Jean-Roger a connu plusieurs années d'errance et d'instabilité à différents niveaux : géographique, professionnelle...

La parentalité est également complexe. Cette difficulté est liée aux ruptures, à une fluctuation ou absence de modèle parental, à l'instabilité de leur relation à l'objet primaire, qui se trouvent réactivées dans leur nouveau statut parental. Les quatre femmes rencontrées ont connu des séparations avec leurs conjoints. Maryse a eu un enfant avec un homme qui lui a fait subir des violences conjugales pendant quatre ans. Par la suite, elle a rencontré un autre homme qui l'a quittée durant sa grossesse et elle aura ensuite deux enfants avec un troisième homme avec lequel elle s'est mariée deux fois, puisqu'elle a divorcé de lui entre-temps. "La violence elle se perpétue ", admet Marie-Françoise. Pour Maryse et Marie-Claire, la violence conjugale prend ainsi le relais de la violence de leur mère adoptive et des agressions sexuelles subies chacune par leur père adoptif. Pour l'une, le conjoint est violent. Pour l'autre, la violence est là envers les enfants, qui seront alors placés à l'ASE (Aide sociale à l'enfance).

Des années après, retrouver ses traces et des liens à la Réunion s'avère nécessaire. Mais ces retrouvailles réactivent les douleurs anciennes de part et d'autre. 
C’est lorsqu'elle a 30 ans que Marie-Claire découvre son histoire : "Je vois que j'ai une mère, que je ne suis pas orpheline de père et de mère. Puisque ma mère avait signé... ce papier pour que je puisse venir en France... faire mes études. Ce qui veut dire que St Clar était au courant que j'étais pas orpheline. Je peux devenir orpheline du jour au lendemain alors que ma mère a signé. Et mon père ne voulait pas que je vienne. Il y a un pouce... C'est-à-dire que lui il avait écrit : "Je ne veux pas que ma fille parte. " Alors pourquoi il y a un pouce ? À la préfecture, à la mairie de St Denis. Ma cousine s'est chargée à me mettre en relation avec ma mère. Et c'est là, ma mère se sentant coupable, a mis un an et demi avant de me répondre. Elle avait honte. Donc c'est moi qui suis allée à sa rencontre en 1987. [...] Moi j'avais du mal à aller voir ma mère. Je voulais pas en fait. Je me dis : "Mais qu'est-ce que je vais lui dire ? Ça va être très très très dur [...]. » C'est quand je suis partie en 1987 qu'elle m'a raconté ce qui s'était passé. Elle m'a dit : «Tu sais, je t'avais retrouvée, la DDASS est venue me voir, m'a fait signer un papier pour que tu ailles à la plaine des Cafres et comme ça le temps que je me refasse une santé et que toi, on puisse te donner une éducation, au bout d'un an, je pouvais venir te rechercher. " Elle poursuit en disant que sa mère aurait voulu récupérer sa fille au bout d'un an mais qu'elle ne s'y trouvait plus.

Maryse va à La Réunion pour la première fois en 1998, elle y retrouve des oncles, tantes, frères et sœurs. Mais ce n'est qu'en 2014 qu'elle comprend avoir été adoptée dans le cadre de la politique de Michel Debré et se rend compte qu'elle n'est pas toute seule à avoir ce vécu. La famille ne lui avait rien révélé. C'est un effondrement. Aujourd'hui, elle continue à apprendre des nouvelles informations sur son passé infantile.

Jean-Paul a séjourné à plusieurs reprises à La Réunion, mais ce n'est que depuis avril 2017 qu'il apprend qu'à l'âge de 3 mois il a été volé à sa mère, puisqu'il prend notamment connaissance d'un document établi par la DDASS dans lequel il est mentionné son abandon par sa mère, mais exsangue de toute signature de sa part.

Le vécu est particulièrement douloureux, et les répercussions psychologiques sont importantes, tant au niveau individuel que dans l'intersubjectivité. Car si enfant, la greffe n'a pas pris en Métropole pour la majorité d'entre eux, quarante ans après, la greffe ne reprend pas à La Réunion, et semble même réactiver les premiers abandons.

\section{LES DIFFICULTÉS RELATIONNELLES INTERGÉNÉRATIONNELLES SONT DONC MASSIVES}

Quand il y a retrouvailles avec les parents biologiques, celles-ci s'avèrent particulièrement difficiles, voire douloureuses parfois. Après quarante ans de silence, d'absence, de mensonges, les retrouvailles sont extrêmement difficiles, voire parfois impossibles avec des non-dits persistants. Du côté des enfants devenus adultes : comment retrouver ses parents ou d'autres membres de sa famille, alors que pendant tant d'années ils se pensaient orphelins ? Avec pour certains, la croyance qu'ils avaient été abandonnés par leurs parents.

Pour les parents, ces retrouvailles conduisent à une réactivation des douleurs du passé. Les parents, s'ils sont toujours vivants, ont vieilli, ils ont aménagé leur vie dans l'absence. Le travail de deuil du vol de leur " maternalité " (Racamier et al., 1961) et de leur parentalité se retrouve alors ébranlé, voire effondré. Les plaies du passé se rouvrent à nouveau. 
Ce retour des enfants perdus et souvent oubliés est donc douloureux à accepter. Ces enfants de retour sont devenus des étrangers aux yeux de leur famille biologique et sont parfois perçus comme des revenants. Il s'agit d'un véritable ébranlement familial de part et d'autre, d'autant que ces retrouvailles n'ont pas été préparées. Et si certains ex-enfants réunionnais ont fait la démarche du retour sur l'Île, souvent avec beaucoup d'appréhension, les parents à La Réunion n'étaient pas dans une même synchronicité. Pour certains, la surprise du retour fait alors effraction.

Ce retour a même l'effet d'un après-coup dans l'histoire du peuple réunionnais. Comment intégrer ces migrants de retour ? Comment ces migrants peuvent-ils s'adapter ? D'autant qu'ils ont été dé-nommés, renommés et qu'ils sont acculturés. Car certains souhaitent se réinstaller à La Réunion.

La relation avec les parents adoptifs reste souvent difficile surtout lorsque ces derniers ont participé à la rupture, au silence, et se sont comportés de façon inadaptée. Reste aujourd'hui la question de savoir si ces parents adoptifs avaient connaissance du contexte dans lequel ils adoptaient leurs enfants.

Les difficultés avec leurs propres enfants sont également importantes. L'histoire de ces enfants réunionnais n'a pu être élaborée. Ou bien elle l'est pour certains mais depuis très récemment. Cette histoire se manifestant par l'expression d'objets bruts non métabolisés, leurs enfants sont devenus contenants de l'histoire parentale plutôt que les héritiers (Feldman et al., 2016), d'autant que cette humiliation n'a pas été reconnue par l'État. Ainsi les enkystements du passé parental se transmettent à la descendance sans être transformés. Au niveau de la descendance, j'ai pu identifier une errance d'affiliation pour certains, pour lesquels les parents ont essayé d'être des parents malgré tout, et d'autres pour lesquels la dysparentalité a été telle que leurs enfants ont été pris en charge par l'ASE. L'histoire se répète.

À propos de sa fille, Marie-Françoise me dit : "J'espère le jour où il y aura un événement qui fera nous rapprocher. "Une crise de transmission est vécue par les deux générations. Seul Jean-Paul, pour lequel son adoption s'est effectuée auprès d'un couple bienveillant, ne semble pas connaître ces difficultés avec ses enfants, d'autant qu'il les a impliqués dans ses recherches de filiation.

\section{LES TROUBLES PSYCHIQUES SONT IMPORTANTS}

Ils partagent tous un profond vécu abandonnique. La symptomatologie recouvre un spectre large : instabilité affective et/ou professionnelle, labilité émotionnelle, cauchemars, peur du noir, peur de l'enfermement, hyperactivité, troubles addictifs, tentatives de suicide répétées qui, pour plusieurs d'entre elles, ont débuté à l'adolescence et sont souvent suivies de séjours en psychiatrie, avec une impression de ne jamais être à sa place (" Jamais à sa place ", "où je dois être ? ", dit Marie-Françoise), des angoisses, de l'anxiété, n'être bien nulle part, dépression, des troubles somatiques tels que la rectocolite-hémorragique, psoriasis. 
Depuis quelques années, chaque retour en Métropole d'un séjour passé sur l'Île de La Réunion, chaque retour d'une assemblée générale des ex-mineurs réunionnais génère pour beaucoup d'entre eux un état de grande vulnérabilité : dépression, tentatives de suicide, errance. Ainsi des fragilités importantes adviennent à chaque rupture, nécessitant un réaménagement psychique : adolescence, maternité, séparation avec le conjoint, adolescence de son enfant,... retrouvailles avec La Réunion, retour en Métropole,...

On sait que la filiation se forme par des liens et des continuités, mais aussi par des séparations et des discontinuités (Konicheckis, 2001). La qualité de l'élaboration psychique et fantasmatique peut rendre ces discontinuités vivables et acceptables, ou au contraire traumatisantes et déliantes. Or, quand il y a effraction psychique, comme c'est le cas pour ces enfants, et que le processus de développement s'inscrit dans l'inélaborable et la déliaison, ces discontinuités sont alors désorganisatrices. Ainsi, chaque rupture agit comme un après-coup et conduit parfois à une décompensation.

Marie-Françoise : «Je suis restée cinq mois à la Réunion. Je suis revenue en France... cassure... hospitalisée en psychiatrie [...] À chaque fois que je reviens de La Réunion, à chaque fois je suis déboussolée. Ils m'ont donné un traitement mais à chaque fois les antidépresseurs, xanax, stillnox..."

Marie-Claire déclenche une rectocolite-hémorragique à l'âge de 20 ans quand elle quitte St Clar. Régulièrement, elle passe par des périodes de dépression notamment après la lecture de son dossier envoyé de St Denis et après la réalisation du film sur son histoire auquel elle a participé. En août 2015, elle dit avoir eu une prescription d'antidépresseurs, elle dit en avoir pris pendant quatre jours, mais a arrêté car elle se sentait "comme un zombie".

L'éjection des enveloppes culturelle (Feldman, 2016), familiale et géographique, à laquelle s'ajoutent bien souvent des faits de violence physique et psychologique, conduit à la question de la légitimité d'existence jamais résolue.

Ces ruptures, ces traumas sont renforcés par l'absence encore actuelle de la reconnaissance de leurs souffrances. Un procès pour la reconnaissance a été intenté en 2001 contre l'État par les enfants réunionnais de la Creuse, à l'initiative de Jean-Jacques Martial.

Un rapport de l'Inspection générale des affaires sociales (IGAS) conclura en 2002 que la migration des pupilles réunionnais avait " globalement respecté la législation en vigueur ". L'affaire d'État que constitua potentiellement ce procès n'a jamais pu éclater faute de soutien. Le procès a été perdu en 2012 après des années de vaines procédures conclues par une décision expéditive de la Cour européenne de justice. Le 18 février 2014, l'Assemblée nationale propose une résolution de loi dans laquelle elle demande à ce que la connaissance historique de cette affaire soit approfondie et diffusée, considérant que l'État a manqué à sa responsabilité morale envers ces pupilles. Ainsi, une commission d'expertise dirigée par Philippe Vitale est mise en place en février 2016 pour travailler à un rendu au gouvernement de propositions d'accompagnement aux ex-mineurs de la Réunion, en 2018. Un point d'étape a déjà été remis en février dernier. 


\section{DISCUSSION}

Ces événements subis depuis le départ de La Réunion, jusqu'à aujourd'hui fabriquent des "traumatismes cumulatifs ", définis par Masud Khan comme l'accumulation de défaillances répétées de l'objet maternel dans son rôle de "barrière protectrice et de moi auxiliaire " $(1976$, p.74). Ils ont un impact sur le développement de l'enfant et son devenir adulte (Feldman, 2013). Au-delà de la désaffiliation mise en œuvre par l'Assistance publique puis la DDASS, c'est d'un abus de filiation dont il s'agit, opérant selon un processus de désubjectivation, et qui a mené à une suspension de leur identité.

\section{LA DÉ-FILIATION ET LA DÉSAFFILIATION}

Pour la sécurisation psychique, les problématiques de filiation et d'affiliation sont centrales dans la construction de l'enfant. En effet, la naissance d'un enfant l'inscrit dans une généalogie, une histoire et une géographie. En tant qu'il est enfant de son père et de sa mère, il est inscrit nécessairement dans ses filiations : paternelle et maternelle, aux familles qui le portent et, au-delà, à ses groupes d'appartenance. D'où l'importance de l'affiliation, comme socle qui préexiste à tout être humain, avant même sa naissance.

Nous utilisons ici la notion de filiation telle qu'elle a été définie par Michael Housemman (citée par Jean Guyotat, 1995), dans une perspective anthropologique. Selon lui, la filiation est régie par un principe généalogique « régissant l'appartenance à une certaine collectivité constituée par des personnes tenues pour issues d'un ancêtre commun " (p.8). Jean Guyotat (ibid.) distingue trois aspects inhérents au lien de filiation. Il évoque la filiation instituée, celle de la transmission du nom dans la relation à autrui, telle que la définit la forme sociale. Ce mode de filiation prend en compte la discontinuité dans l'ordre des générations. Il y aurait une filiation métaphorique et une filiation métonymique. Entre le corps du père, qui donne son nom, et le corps de l'enfant, qui le reçoit, il y aurait un transfert de signification, une "métaphore ". Alors qu'entre le corps de la mère qui contiendra en elle le corps de l'enfant, il s'agirait d'une filiation de corps à corps "métonymique ", un lien de sang, un lien de continuité. Enfin, la filiation narcissique concerne le processus d'affiliation. Le lien "s'inscrit [alors] dans la notion de groupe de filiation qui suppose la reduplication du même de génération en génération, à partir d'ancêtres prestigieux " (p.38). Étant donné que chaque sujet se voit assigné à une place et une mission dans le groupe auquel il appartient (famille, groupe social), la filiation l'assujettit à la chaîne générationnelle.

Le terme d'affiliation est emprunté à l'anthropologie : il s'agit des appartenances à des groupes (Levi-Strauss, 1973, p.132), qu'ils soient culturels, sociaux, ethniques, religieux,... Les affiliations sont plurielles et, comme la filiation, ces deux processus interactifs supposent une élaboration constante du sujet tout au long de son développement et des différents âges de la vie. Filiation et affiliation permettent ensemble de tisser l'inscription de l'individu dans la continuité des générations. 
La filiation se définit aussi par le nom qui donne une légitimité d'existence, puisqu'il a une origine, une fonction, un sens. Le nom apporte un sentiment de continuité d'existence à l'individu inévitablement pris dans des expériences de discontinuité et de rupture. Il représente une liaison à l'intérieur même du psychisme qui permet de définir la fonction narcissique de la paternité, qui y préserve non seulement la continuité de l'espèce mais aussi celle de l'individu. Ainsi, changer de nom, c'est modifier intentionnellement le destin de l'enfant.

Enfin, l'affiliation liant le sujet au social est régie par les termes d'un contrat "narcissique " dans le sens où il doit être investi narcissiquement par les deux parties contractantes, le sujet et son groupe (CastoriadisAulagnier, 1975). En l'absence d'investissement narcissique, l'enfant se retrouve "en suspens ». Et la violence qu'il subit se situe à l'endroit où on lui inflige de renoncer à tout ce qui constituait son identité d'avant.

LES EFFETS INDIVIDUELS ET TRANSGÉNÉRATIONNELS D'UNE POLITIQUE DE DÉSUBJECTIVATION

L'exposition à ces multiples ruptures géographiques de filiation et d'affiliation a été destructrice et a amené à une mise en péril de leurs "fondements généalogiques" (Ayoun et Tabone, 1994, p.28). Ainsi, les enfants réunionnais ont subi une métamorphose identitaire, difficile voire impossible à assumer. De plus, cette désaffiliation a engendré une faille narcissique primaire qui, à un moment ou à un autre, bloque le sujet dans son fonctionnement psychique ou l'entraîne vers " un effondrement identitaire des plus sévères, qu'un travail analytique n'est pas assuré d'enrayer " (Allouch, 2001, p.38).

Cette rupture de filiation a été aggravée par l'éclatement de la fratrie puisque frères et sœurs ont souvent été délibérément séparés et ont ainsi été nommés différemment. Le but était donc bien de briser une histoire commune. Cette rupture a également été accentuée par les mensonges faits aux parents et aux enfants. Alors que le secret a été un des principes de l'institution de la protection de l'enfance de l'époque, il n'en a pas été de même du camouflage de la vérité. Le mensonge est ce qui est le plus dévastateur pour l'enfant puisqu'il se construit avec cette croyance en la protection des adultes. Le mensonge comme modalité relationnelle avec les enfants conduit à une perte de confiance des enfants en l'adulte, c'està-dire une perte de ses "théories de la vie " (Bailly, 2007). Ces "théories " (p.241) désignent un sous-ensemble du champ symbolique, c'est-à-dire qu'au cours des premières années de la vie, un enfant travaille à sa construction et fera des hypothèses sur ses origines, les différences de sexe, la parenté, le rôle des parents... Les traumatismes viennent ainsi bousculer sans cesse ces théories que l'enfant construit au cours de son développement. Son historicité est alors remise en question.

L'abus de filiation se situe ainsi dans le zèle qu'ont eu les responsables et les assistantes sociales de la DDASS à œuvrer dans le cadre d'une opération politique. Pour sa part, Jablonka (2007, p.227) évoque un détournement des dispositifs de protection de l'enfance par l'État, pour imposer une nouvelle culture à ces enfants. Enfin, Ascaride \& al. 
(2004) mentionnent l'attitude du directeur du foyer de Guéret de l'époque, qui, observant le mal-être de ces enfants, voulait les ramener à La Réunion le temps des vacances. Il raconte avoir envoyé un courrier à Michel Debré mais il a essuyé un refus catégorique. Réunionnais luimême, il a injecté de la culture créole au sein du foyer : repas créoles, musique de séga... Quelque temps plus tard, ce directeur a été démis de ses fonctions.

Cet abus de filiation subi résonne avec celui vécu par les ascendants. En effet, les enfants réunionnais ont comme vécu un après-coup de l'histoire de leurs ascendants colonisés, esclaves sur une durée de deux-cents ans : ceux-là mêmes étaient assujettis à leurs maîtres, avaient l'obligation de porter le nom de leurs propriétaires (Payet, 2001) et devaient pratiquer le catholicisme, seule religion autorisée (articles du Code noir). Les enfants réunionnais transférés en Métropole désaffiliés, "adoptés ", ont également dû porter le nom de leurs "nouveaux parents " métropolitains, être baptisés et suivre le catéchisme dans un contexte spécifique. Beaucoup ont été exposés à des violences. Il s'agit donc d'une "double peine " et ils sont victimes d'un "double déni ": leur héritage familial est constitué d'humiliations déniées par le politique, humiliations qu'ils ont à nouveau vécues dans la réalité, et déniées jusqu'à aujourd'hui encore. Malika Mansouri (2013, p.165) rappelle l'analyse critique de Frantz Fanon face à l'école culturaliste d'Alger, questionnant notamment les conséquences psychologiques individuelles délétères d'une situation de domination, telle qu'elle a été notamment à l'œuvre durant la colonisation. En effet, Fanon (1952) parle d'aliénation du colonisé.

\section{CONCLUSION}

Ces effets de cet exil institué d'enfants nous contraignent à penser notre clinique. Celle-ci doit nécessairement prendre en compte l'intrapsychique mais également l'intersubjectif et le collectif. Afin d'apporter un soin suffisamment bon, il est aujourd'hui nécessaire d'agir à deux niveaux : collectif et individuel.

D'une part, il est fondamental que l'État français reconnaisse que 2015 enfants ont été transférés en Métropole prioritairement dans une intentionnalité politique plutôt que pour répondre à l'intérêt des enfants, même si la concordance a été recherchée. Si l'État ne peut pas remettre en question les principes de fonctionnement de l'Assistance publique puis la DDASS qui, à l'époque, avait comme mission de gérer le flux d'enfants (Feldman \& Hazan, 2017), il peut néanmoins reconnaître le passage à l'acte qu'est cette transplantation organisée sous l'égide du mensonge ainsi que ses effets dévastateurs sur les individus eux-mêmes, et sur la descendance, dans ce que j'ai pu en percevoir. Il s'agira ainsi, au niveau collectif et individuel, de réparer narcissiquement et juridiquement les atteintes portées à l'identité et la filiation. La problématique de la transmission et des secrets étant déjà au cœur de l'histoire des Réunionnais (Payet, 2001), cette transplantation des enfants opère ainsi comme un après-coup de l'histoire du 
peuple réunionnais, histoire constituée de ruptures, de conflits, de métissages, de la colonisation et de l'esclavage.

Au niveau individuel, la prise en charge psychologique devra prendre en compte cette réalité historique, politique et institutionnelle (Feldman \& Mansouri, 2018). Reconnaître les troubles psychiques sans considérer le contexte ne ferait que renforcer les symptômes et leur transmission à la descendance. Dans cette articulation du collectif et de l'individuel, il sera alors utile pour le thérapeute de faire fonction d'hébergement psychique de reconnaissance.

\section{RÉFÉRENCES}

Allouch, E. (2001). Le mal des origines. Cliniques méditerranéennes, 64, 29-40.

Ascaride, G., Spagnoli-Bègue, C. \& Vitale, Ph. (2004). Tristes tropiques de la Creuse. Romainville : K'A.

Ayoun, P. \& Tabone, B. (1994). L'attaque de filiation : une forme de maltraitance. Synapse, 108, 27-31.

Bailly, L. (2007). Métapsychologie de la traumatisation. Revue francophone du stress et du trauma, 7(4), 239-243.

Castoriadis-Aulagnier, P. (1975). La violence de l'interprétation. Du pictogramme à l'énoncé. Paris : Puf.

Devereux, G. (1972). Ethnopsychanalyse complémentariste. Paris : Flammarion, 1985.

Fanon, F. (1952). Peau noire, masques blancs. Paris : Seuil.

Feldman, M., Mouchenik, Y. \& Moro M.R. (2008). Les enfants juifs cachés en France pendant la Seconde Guerre mondiale : des traces du traumatisme repérables plus de soixante ans après. La Psychiatrie de l'enfant, 51(2), 481-513.

Feldman, M. (2009). Entre trauma et protection : quel devenir pour les enfants juifs cachés en France (1940-1944) ? Toulouse : Érès.

Feldman, M. (2013). Attaques cumulées des liens de filiation et d'affiliation : quel devenir pour l'enfant ? Cliniques méditerranéennes, 88, 249-263.

Feldman, M. (2016). Une parentalité ébranlée par les violences collectives. Dans A. Ciccone (dir.), Violences dans la parentalité (p.119-127). Paris : Dunod.

Feldman, M., Mansouri, M., Revue, P. \& Moro, M.R. (2016). Une clinique des affiliations pour une psychopathologie contemporaine. La Psychiatrie de l'enfant, 59(1), 291-307.

Feldman, M. \& Hazan, K. (2017). Histoires secrètes. Les enfants juifs et l'Assistance publique. Paris : In Press.

Feldman, M. \& Mansouri, M. (2018). Une clinique du colonial : panser les aprèscoups. Le Coq héron, 234 (sous presse).

Gampel, Y. (2006). La honte dans le contexte de violence sociopolitique. Le Coq héron, 184, 68-75.

Guyotat, J. (1995). Filiation et puerpéralité. Logiques du lien. Paris : Puf.

Jablonka, I. (2006). Ni père ni mère. Histoire de l'Assistance publique (1874-1939). Paris : Le Seuil.

Jablonka, I. (2007). Enfants en exil. Transfert de pupilles réunionnais en Métropole (1963-1982). Paris : Le Seuil.

Khan, M. (1976). Le concept de traumatisme cumulatif. Dans Le soi caché (p.69-99). Paris : Gallimard.

Konicheckis, A. (2001). Paradoxes et fonctions narcissiques de la filiation. Cliniques méditerranéennes, 63,143-166.

Levi-Strauss, C. (1973). Organisation sociale. Dans Anthropologie structurale deux (p.89-135). Paris : Plon, 2010. 
Mansouri, M. (2013). Révoltes postcoloniales au cour de l'Hexagone. Paris : Puf.

Moro, M.R. (1994). Parents en exil. Psychopathologie et migrations. Paris : Puf.

Payet, G. (2001). Nom et filiation à La Réunion : de l'histoire à la clinique. Cliniques méditerranéennes, 63, 179-192.

Racamier, P.-C., Sens, S. \& Carretier, L. (1961). La mère, l'enfant dans les psychoses du post-partum. L'Évolution psychiatrique, 26(4), 525-570.

Verdier, P. (1978). L'enfant en miettes. Paris : Dunod, 2004.

Winnicott, D.W. (1984). Les enfants et la guerre. Paris : Payot, 2004.

Marion Feldman

UFR Sciences Psychologiques et Sciences de l'Education (SPSE)

200 avenue de la République

92000 Nanterre

marion.feldman@free.fr 